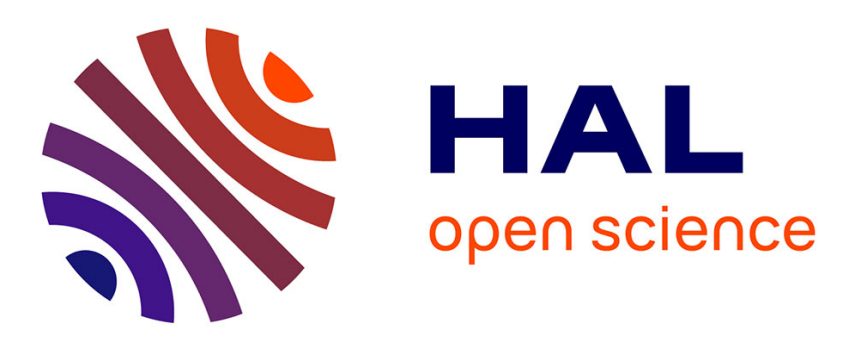

\title{
Reconstruction of Coronary Arteries from a Single Rotational X-Ray Projection Sequence
}

Christophe Blondel, Grégoire Malandain, Régis Vaillant, Nicholas Ayache

\section{To cite this version:}

Christophe Blondel, Grégoire Malandain, Régis Vaillant, Nicholas Ayache. Reconstruction of Coronary Arteries from a Single Rotational X-Ray Projection Sequence. IEEE Transactions on Medical Imaging, 2006, 25 (5), pp.653-663. 10.1109/TMI.2006.873224 . inria-00614993

\section{HAL Id: inria-00614993 https://hal.inria.fr/inria-00614993}

Submitted on 17 Aug 2011

HAL is a multi-disciplinary open access archive for the deposit and dissemination of scientific research documents, whether they are published or not. The documents may come from teaching and research institutions in France or abroad, or from public or private research centers.
L'archive ouverte pluridisciplinaire HAL, est destinée au dépôt et à la diffusion de documents scientifiques de niveau recherche, publiés ou non, émanant des établissements d'enseignement et de recherche français ou étrangers, des laboratoires publics ou privés. 


\title{
Reconstruction of Coronary Arteries from a Single Rotational X-Ray Projection Sequence
}

\author{
Christophe Blondel, Grégoire Malandain, Régis Vaillant and Nicholas Ayache
}

\begin{abstract}
Cardiovascular diseases remain the primary cause of death in developed countries. In most cases, exploration of possibly underlying coronary artery pathologies is performed using X-ray coronary angiography. Current clinical routine in coronary angiography is directly conducted in 2-D projection images from several static viewing angles. However, for diagnosis and treatment purposes, coronary artery reconstruction is highly suitable. The purpose of this study is to provide physicians with a 3-D model of coronary arteries, e.g. for absolute threedimensional measures for lesion assessment, instead of direct projective measures deduced from the images, which are highly dependent on the viewing angle. In this article, we propose a novel method to reconstruct coronary arteries from one single rotational X-ray projection sequence. As a side result, we also obtain an estimation of the coronary artery motion. Our method consists of 3 main consecutive steps: (1) 3-D reconstruction of coronary artery centerlines, including respiratory motion compensation, (2) coronary artery 4-D motion computation, and (3) 3-D tomographic reconstruction of coronary arteries, involving compensation for respiratory and cardiac motions. We present some experiments on clinical datasets, and the feasibility of a true 3-D Quantitative Coronary Analysis is demonstrated.
\end{abstract}

Index Terms-Angiocardiography, Coronarography, Image Motion Analysis, Image Reconstruction, Tomography

\section{INTRODUCTION}

A Ccording to the World Health Organization [1, page 48], coronary heart disease is the major cause of death worldwide. In particular, coronary artery lesions are involved in most cases of heart failure and are thus the subject of medical imaging examinations when a pathology is suspected.

Currently, clinical routine relies on direct analysis of Xray coronary angiographies acquired from several static acquisitions from distinct viewing angles. It thus produces 2-D measures (e.g. QCA - Quantitative Coronary Analysis) which suffer from well known viewing angle dependence, magnification factor, and superimposition effects. However, to achieve adequate therapeutic orientation, 3-D measures such as absolute vessel cross-sectional area would be of interest as they can be used to specify diameter and length of angioplasty balloons or stents to be used. In this context, three-dimensional reconstruction of coronary arteries would be of great clinical and diagnostic interest as it would provide physicians with 3-D absolute measures. Our purpose is thus to obtain tomographic reconstructions of coronary arteries, in a CT-like

Christophe Blondel is with General Electric HealthCare, Buc, France and with Chir and Epidaure teams at INRIA, Sophia-Antipolis, France. Grégoire Malandain and Nicholas Ayache are with Epidaure team. Régis Vaillant is with General Electric HealthCare.

This work was supported by Association Nationale de la Recherche Technique (ANRT) and General Electric HealthCare. manner, from the most widely available imaging modality for coronary artery examination, X-ray coronary angiography.

Such 3-D information may be obtained by biplane angiography, since this modality provides two (almost) synchronized projections of the coronary arteries [2]-[4], or by selecting two views from two single-plane angiograms [5]. However, using only two projections is not sufficient to provide a precise measure of cross-section areas. Reconstructing the coronary arteries still remains a very challenging task, despite recent advances in medical imaging hardware and methodologies. In $\mathrm{X}$-ray coronary angiography, the introduction of the digital flat panel [6] combined with a rotational acquisition mode [7] allowed for the proposal of new techniques in coronary artery modeling. The number of projections used for reconstruction can be increased by selecting the ones that correspond to the same cardiac time in a rotational acquisition, as in [8], [9], but most of the acquired images are discarded in such a procedure. By selecting projections close to a cardiac time, the number of used projections increases [10] but reconstruction artifacts may appear due to motion, and a significant number of acquired images are still discarded.

The main two difficulties that arise for the tomographic reconstruction of coronary arteries from angiograms are indeed the respiratory and cardiac motions that are visible in the Xray projection sequence. In this article, we present a study that demonstrates the feasibility of the reconstruction of a 3$\mathrm{D}$ image of the coronary arteries from $a$ single rotational X-ray projection sequence, without requiring any additional measure (e.g. ECG).

Contrary to iterative methods that alternate between motion estimation and tomographic reconstruction (e.g. [11]), the proposed method is direct and consists of three major steps (see Fig. 1): (1) static 3-D reconstruction of coronary artery centerlines at one given cardiac phase, (2) estimation of 4-D motion from resulting set of 3-D lines, and (3) 3D tomographic reconstruction of coronary arteries performed by integrating cardiac motion compensation. This last step is only sketched here, details can be found in [12].

The remainder of this article is organized as follows. In Section II, we describe the rotational acquisition protocol that was used and the datasets we were provided with. Sections III, IV, and V detail, respectively, the 3-D centerlines reconstruction (which is coupled with respiratory motion correction), the coronary artery motion estimation, and finally the tomographic reconstruction with motion compensation. Experiments on patient datasets are presented in Section VI, while the next Section VII discusses the proposed method and some potential clinical applications. Section VIII gives some perspectives. 


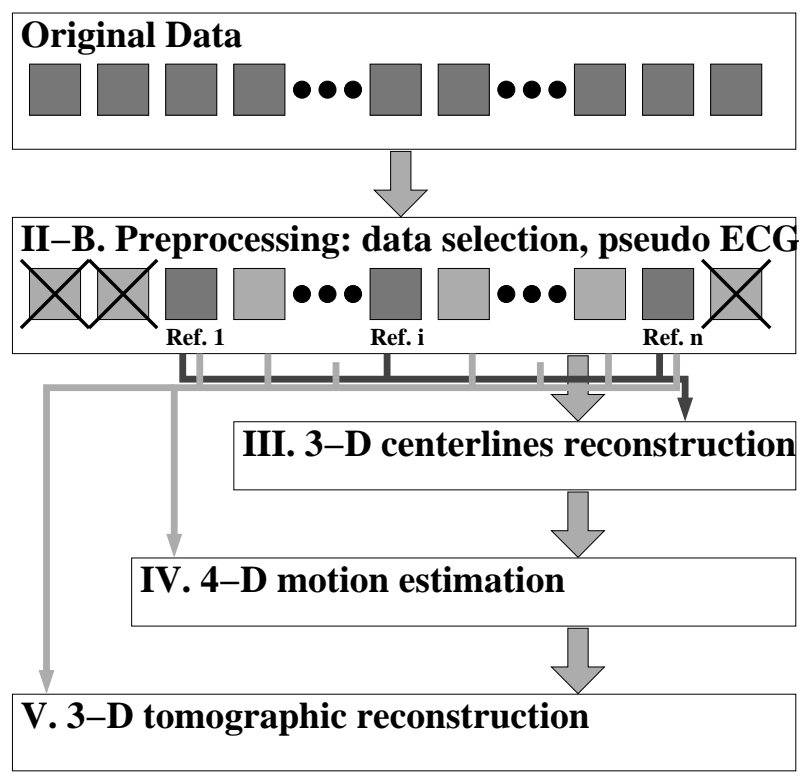

Fig. 1. Overview of the proposed method. The reference images (images that are synchronous w.r.t. the cardiac cycle) are identifi ed in a pre-processing stage, and some data, at the extremities of the temporal sequence, are discarded. These reference images are used to reconstruct a static 3-D centerlines model at the reference time. This model and all the data allow the calculation of a 4-D deformation fi eld that estimates the cyclic heart motion. This 4-D motion and all the data (from ref. 1 to ref. n) are fi nally used to provide the tomographic reconstruction of a 3-D image of the coronary arteries.

\section{DATA AND PRE-PROCESSING}

\section{A. Data}

Images were acquired on an Innova 2000 system, from General Electric HealthCare, which is equipped with a digital flat panel detector. The gantry performs a rotation while acquiring the images [13]. The gantry motion is characterized by constant SID (Source Intensifier Distance) value, constant CRA/CAU (Cranio/Caudal) angle value, and varying LAO/RAO (Left/Right Anterior Oblique) angle. Thus, the rotation occurs in patient axial plane, with maximum LAO/RAO angle amplitude of $200^{\circ}$. Top rotation speed is $40^{\circ} \mathrm{s}^{-1}$, leading from 3 to 5 seconds long acquisitions. Angiograph acquisition frame rate is $30 \mathrm{~Hz}$. Thus, this protocol provides us with the imaging of 3 to 7 cardiac cycles. Images acquired at the same cardiac phase are approximately separated by a $30^{\circ}$ angular shift, depending on patient heart rate.

For each patient, we have a single rotational sequence consisting of $\tilde{\mathcal{O}}$ images $I_{n}$ with spatial resolution $768 \times 768$ pixels and pixel size of $0.2 \mathrm{~mm}$. We resampled these images into a $512 \times 512$ lattice for computational purposes. In addition, a precalibration step allowed to estimate the geometrical acquisition parameters that are summarized in $\mathcal{O}$ projection matrix applications $\mathbf{M}_{n}: \mathbb{R}^{3} \longrightarrow \mathbb{R}^{2}$, the matrix $\mathbf{M}_{n}$ being associated to image $I_{n}$.

\section{B. Preprocessing}

A prerequisite for our method is the identification of a pseudo-cardiac time (or observed cardiac phase) for all images in the sequence. This information is computed solely from the image sequence information, without any external measures such as ECG signal. The basic idea is the following: along the cardiac cycle, systole is characterized by myocardium contraction and a global top-to-bottom motion of the coronary tree in the axial direction, while diastole is characterized by myocardium relaxation and a global bottom-to-top motion of the coronary tree in the axial direction. In addition to the cardiac motion, the coronary arteries are also subject to the respiratory motion that consists mainly in a vertical translation in the axial direction, but of much lower frequency than the cardiac one. We then assumed that the high frequency part of the global vertical motion of coronary arteries in the image sequence is directly related to the cardiac phase.

To identify the vertical component of motion in the image sequence, we first compute for all images $I_{n}(x, y)$ the vector of horizontal line integrals $H_{n}(y)=\sum_{x} \bar{I}_{n}(x, y)$ of an associated local contrast image $\bar{I}_{n}(x, y)$ (obtained by applying a morphological top-hat operator [14] on the initial image $I_{n}$ ) over the horizontal coordinate. The vertical motion between two successive frames is estimated by identifying the shift along the vertical axis that minimizes the sum of squared differences between the corresponding $H_{n}$. The process is carried out over the complete sequence and leads to a nearly periodic signal over time, whose high frequency characterizes the heart beat. Quasi synchronous images can be easily identified by either selecting image indices at local maxima of the integral signal, located at end-diastole, or selecting image indices at local minima of the integral signal, located at end-systole [41]. In practice, quasi synchronous images acquired at end-diastole are preferred because they correspond to the most relaxed and stable state along heart motion, and consequently reduces superimpositions and potential asynchronism. The selected quasi synchronous images, which correspond to the same cardiac phase, are called reference images. The set of reference images will be denoted by $\mathcal{R}$ and its cardinal by $\tilde{\mathcal{R}}$.

Using reference image indices, we assign to each frame a normalized time that encodes the observed cardiac phase, relatively to cardiac phase in reference images. Normalized times belong to the $[0,1[$ interval. The computation scheme is the following: two successive reference images are respectively assigned to normalized time $t=0$ and normalized time $t=1$, then normalized times of intermediate images are given by linear interpolation.

Time normalization is adapted to cardiac period changes during the acquisition, which is often the case, as contrast agent injection usually accelerates heart motion. Indeed, we do not assume that the number of acquired images between two references times is fixed for a given sequence. However, images before the first and after the last reference images can not be assigned a normalized time. In the following, we discard these images from the sequence to only consider the $\tilde{\mathcal{N}}$ images, $\tilde{\mathcal{N}} \leq \tilde{\mathcal{O}}$, between the first and last references images. The normalized time of image $I_{n}$ is denoted $t_{n}$.

\section{3-D CENTERLINES RECONSTRUCTION}

The first stage of our method is the reconstruction of 3D centerlines from the reference images, corresponding to 


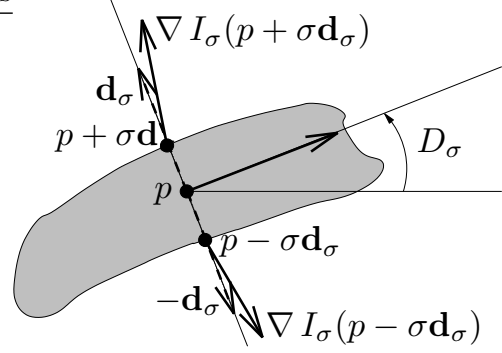

Fig. 2. Filter response computation scheme. Filter response at pixel $p$ is computed by calculating an edge response on both sides of the potential rectilinear structure, at distance $\sigma$ from pixel $p$ in direction $\mathbf{d}_{\sigma}$ orthogonal to the structure direction $D_{\sigma}$.

normalized time $t=0$. These images are selected from one single sequence and acquired at the same cardiac phase but from distinct viewing angles. Thus, they are supposed to be uncorrupted by cardiac motion, but are subject to respiratory motion. The extraction of the coronary artery centerline in 2-D images and their 3-D reconstruction, including the respiratory motion compensation, is detailed below.

\section{A. Vessel enhancement}

Our first prerequisite is to enhance the vessels in the angiograms. We used the approach first proposed in [15], [16] and extended in [17]. It relies on a multiscale Hessian-based filtering that enhances curvilinear structures.

For a given scale $\sigma$, an original image $I$ is first convoluted with a 2-D Gaussian $G_{\sigma}$ with standard deviation $\sigma$. The convoluted image is denoted by $I_{\sigma}=I * G_{\sigma}$. The Hessian matrix of the convoluted image is computed by:

$$
\mathbf{H} I_{\sigma}=\left(\begin{array}{cc}
\frac{\partial^{2} I_{\sigma}}{\partial x^{2}} & \frac{\partial^{2} I_{\sigma}}{\partial y \partial x} \\
\frac{\partial^{2} I_{\sigma}}{\partial x \partial y} & \frac{\partial^{2} I_{\sigma}}{\partial y^{2}}
\end{array}\right)
$$

where the second derivatives of $I_{\sigma}$ are calculated by convoluting $I$ with the corresponding second derivatives of $G_{\sigma}$. The eigenvalues and eigenvectors of the Hessian allow to characterize the local structures [18]. It follows that the direction $D_{\sigma}$ of a potential locally rectilinear structure, i.e. a vessel, can be estimated by $\tan \left(2 D_{\sigma}\right)=2 \frac{\partial^{2} I_{\sigma}}{\partial x \partial y} /\left(\frac{\partial^{2} I_{\sigma}}{\partial x^{2}}-\frac{\partial^{2} I_{\sigma}}{\partial y^{2}}\right)$.

Let $\mathbf{d}_{\sigma}$ be an unitary vector orthogonal to direction $D_{\sigma}$. A vessel point should exhibit strong edge information (the vessel borders) at some distance in both directions $\mathbf{d}_{\sigma}$ and $-\mathbf{d}_{\sigma}$. We evaluate this edge information as the derivative of $I_{\sigma}$ with respect to $\mathbf{d}_{\sigma}$ at a distance $\sigma$, and we end up with a filter designed for rectilinear structures:

$$
R_{\sigma}(p)=\min \left\{\nabla I_{\sigma}\left(p+\sigma \mathbf{d}_{\sigma}\right) \cdot \mathbf{d}_{\sigma},-\nabla I_{\sigma}\left(p-\sigma \mathbf{d}_{\sigma}\right) \cdot \mathbf{d}_{\sigma}\right\} .
$$

Figure 2 shows an illustration of the filter response computation scheme. This filter enhances rectilinear structures with width close to scale $\sigma$. Moreover, it also has maximum response at vessel center. Since the observed vessels have highly varying sizes, the previous computation is extended to multiple scales and conducted for a set of scales $\Sigma$, adapted for smallest to largest vessels. In pixels length unit, for $512^{2}$ spatial resolution images, we use $\Sigma=\{1,2,3,4,5,6\}$. At each point $p$, the best scale $\sigma^{\star}(p)$ is selected according to the maximum filter response: $\sigma^{\star}(p)=\arg \max _{\sigma \in \Sigma} R_{\sigma}(p)$.

To compare and normalize the filter responses across scales, the concept of $\gamma$-derivatives was used [17], [19]. The direction and the response associated with the best local scale are collected into a multiscale direction $D^{\star}$ and a multiscale filter response map $R^{\star}$. This multiscale filter response $R^{\star}$ can be considered as a likelihood for pixels to belong to the projected centerline of a coronary artery.

\section{B. 2-D centerlines extraction}

From the above computed multiscale responses, we now build a set of 2-D curves that represent the coronary artery centerlines. This will be done in three steps: subpixel local directional maxima computation, hysteresis thresholding of local directional maxima, and points linking.

1) Subpixel local directional maxima extraction: $R^{\star}$ exhibits higher intensities in the center of a rectilinear structure than in the vicinity of its borders. A pixel $p$ is then likely to belong to the vessel centerline if $R^{\star}$ is maximal at $p$ along a direction orthogonal to the vessel. Such a direction is given by $\mathbf{d}_{\sigma^{\star}}(p)$, so pixel $p$ is a local directional maximum if the following conditions hold: $R^{\star}(p)>R^{\star}\left(p+\mathbf{d}_{\sigma^{\star}}\right)$ and $R^{\star}(p)>R^{\star}\left(p-\mathbf{d}_{\sigma^{\star}}\right)$.

A subpixel estimation of the detected local maxima is achieved by fitting a quadric on points $\left(p-\mathbf{d}_{\sigma^{\star}}, R^{\star}(p-\right.$ $\left.\left.\mathbf{d}_{\sigma^{\star}}\right)\right),\left(p, R^{\star}(p)\right)$, and $\left(p+\mathbf{d}_{\sigma^{\star}}, R^{\star}\left(p+\mathbf{d}_{\sigma^{\star}}\right)\right)$. After some calculations, it turns out that this subpixel maximum $\hat{p}$ is given by

$$
\hat{p}=p+\frac{R^{\star}\left(p-\mathbf{d}_{\sigma^{\star}}\right)-R^{\star}\left(p+\mathbf{d}_{\sigma^{\star}}\right)}{2\left(R^{\star}\left(p+\mathbf{d}_{\sigma^{\star}}\right)+R^{\star}\left(p-\mathbf{d}_{\sigma^{\star}}\right)-2 R^{\star}(p)\right)} \mathbf{d}_{\sigma^{\star}} .
$$

The extracted set of points contains most of the vascular but also many non vascular structures that have to be excluded.

2) Hysteresis thresholding: most of the irrelevant local directional maxima that do not correspond to vascular structures are characterized by a low multiscale filter response and the small size of the connected components they belong to. Hysteresis thresholding is suited to discard them since it offers the possibility to retain only the elements that match both an intensity criterion and a criterion of size of the connected components. Hysteresis thresholding requires both a high and a low thresholds. We compute these thresholds as quantiles of the cumulated histogram of the multiscale filter response maps over the complete sequence. The low threshold is set to a typical value of the multiscale filter response on vessels, while the high threshold is set to a typical value of the multiscale filter response on vessel centerlines. These quantiles can be related to relative area respectively occupied by vessels and vessel centerlines in the images. From our experiments, we chose the $90^{\text {th }}$ percentile for the low threshold and the $98^{\text {th }}$ percentile for the high threshold. In addition, hysteresis thresholding allows to retain only sufficiently large connected components. The minimal connected component size was set to 5 pixels. 


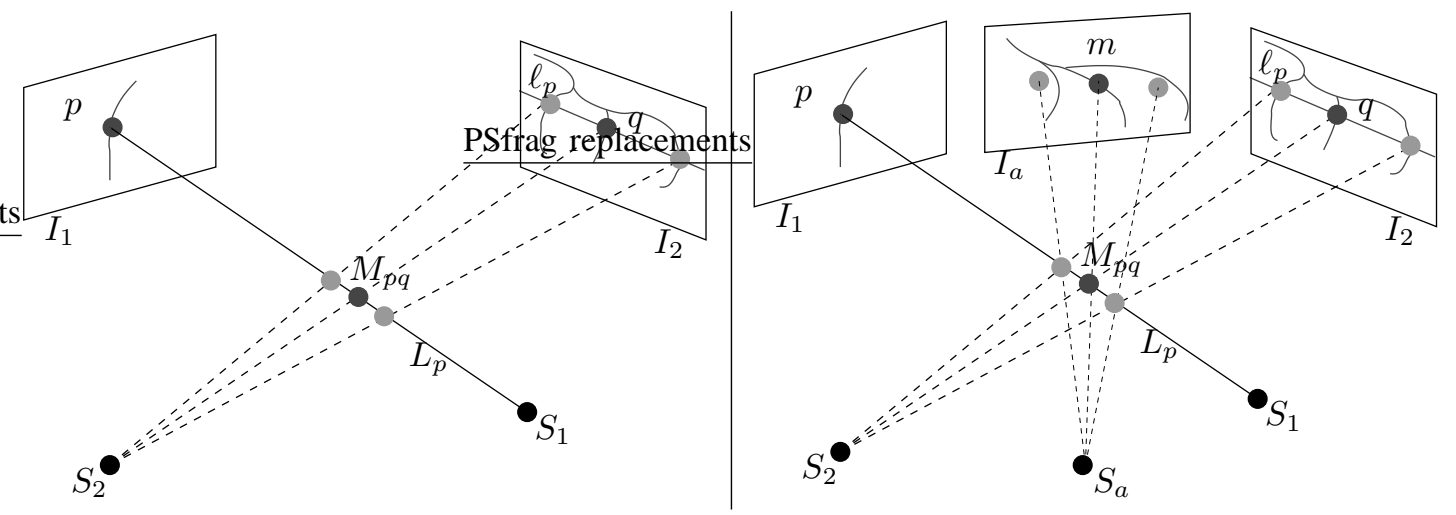

Fig. 3. Epipolar constraint. The 3D point that projects at $p$ in image $I_{1}$ is located on the $3 \mathrm{D}$ line $L_{p}$ (that joins $p$ to $S_{1}$ ). Consequently, its projection in image $I_{2}$ is located on the projection $\ell_{p}$ of the line $L_{p}$. With two images (left), there are many possible 3D points as intersections of $\ell_{p}$ with vessels in $I_{2}$. However, only one of them projects on a vessel in the additional image $I_{a}$ (right). This allows to penalize spurious reconstructed $3 \mathrm{D}$ points.

3) Points linking: local directional maxima have been extracted point-wise, but vessels projected in images have a connected structure. We recover this structure by linking points that belong to the same component using the method described in [20]. We denote by $\mathcal{C}_{I}$ the set of the 2-D connected centerlines extracted from image $I$.

\section{Multi-ocular matching}

Building correspondences between the centerlines of two reference images, $I_{1}$ and $I_{2}$, that are from the same cardiac phase but acquired from two distinct viewing angles, enables the three-dimensional reconstruction of the coronary artery centerlines by applying triangulation and epipolar constraints.

Most of the proposed approaches for 3-D reconstruction of coronary artery centerlines rely on only two angiographic views [21], typically obtained by biplane angiography. The rotational acquisition allows to get between 3 and 7 reference frames, depending on gantry rotation speed and on patient heart rate. We propose to use all the available images to perform a multi-ocular matching of the extracted centerlines in reference images, as basically described in [22]. This is achieved by optimizing a matching criterion detailed below.

1) Asymmetric matching: let us first consider the asymmetric problem of matching a set of linked points, denoted by $C=\left(p_{1}, \ldots, p_{N}\right) \in \mathcal{C}_{I_{1}}$, in a first image $I_{1}$, with the extracted centerline points in a second image $I_{2}$.

$\triangleright$ Problem formulation: As illustrated by Figure 3, a point $p$ in $I_{1}$ is the projection of a $3-\mathrm{D}$ point $M$ located in 3-D line $L_{p}$, joining source position $S_{1}$ to projection position $p$. The projection of the $3-\mathrm{D}$ line $L_{p}$ in $I_{2}$, denoted $\ell_{p}$, must contain the projection $q$ of $M$ in $I_{2}$ : this is the epipolar constraint.

Unfortunately, the epipolar constraint does not generally yield a single match in $I_{2}$ for each $p \in I_{1}$. Indeed, in most cases, line $\ell_{p}$ intersects more that one centerline in $I_{2}$, resulting in a set of matching candidates in $I_{2}$ (see Figure 3, left). Our experiments shows an average of 5 matching candidates per point. Let $\mathcal{Q}_{i}$ denote the set of matching candidates in $I_{2}$ for point $p_{i}$ in $I_{1}$. Building the correspondences for a set of linked points $\left(p_{1}, \ldots, p_{N}\right)$ of $I_{1}$ consists then in choosing a set of points $\left\{q_{1}, \ldots, q_{N}\right\} \in \mathcal{Q}_{1} \times \cdots \times \mathcal{Q}_{N}$.

To compare different matching configuration, we now design a criterion to measure the quality of a given matching hypothesis $\left(p_{i}, q_{i}\right)_{i=1 \ldots N}$. This quality measure is composed of an external energy term, involving reference images information, and an internal energy term, favoring intrinsically coherent matching configurations, that are both detailed hereafter.

$\triangleright$ External energy term: To disambiguate between the matching candidates, the reference images other than $I_{1}$ and $I_{2}$ are used. This additional information is indeed useful: among the several matching candidates given by the epipolar constraint, only one will be coherent with the additional views in most cases. As shown on the right hand side of Figure 3, to each epipolar candidate corresponds a 3-D point using reconstruction by triangulation, whose projection in additional images is on a vessel only for the correct correspondence.

A numerical criterion that reflects the relevance of a reconstructed 3-D point is the value of the multiscale filter response of its projection in the additional views. Let $I_{a} \in \mathcal{R} \backslash\left\{I_{1}, I_{2}\right\}$ be such an additional view (from the same cardiac phase than $I_{1}$ and $I_{2}$ by the definition of $\mathcal{R})$. To any given matching pair $(p, q)$ corresponds a 3-D reconstructed point $M_{p q}$, whose projection in additional image $I_{a}$ is $m=\mathbf{M}_{a} M_{p q}$. We recall that $R_{a}^{\star}$ is the multiscale filter response map associated to the additional image of index $a$. The external energy term measuring matching pair $(p, q)$ quality is defined by:

$$
A M_{\mathrm{Ext}}(p, q)=\frac{1}{\tilde{\mathcal{R}}-2} \sum_{I_{a} \in \mathcal{R} \backslash\left\{I_{1}, I_{2}\right\}} R_{a}^{\star}\left(\mathbf{M}_{a} M_{p q}\right)
$$

where $\tilde{\mathcal{R}}$ stands for the number of reference images.

This criterion reaches high values for matching pairs that are coherent with additional reference images.

$\triangleright$ Internal energy term: the above criterion is convenient for points, but does not take into account the intrinsic linked structure of vessel centerlines. Indeed, a linked set of points in image $I_{1}$, that represents a detected centerline, is more likely to project as a single connected component 

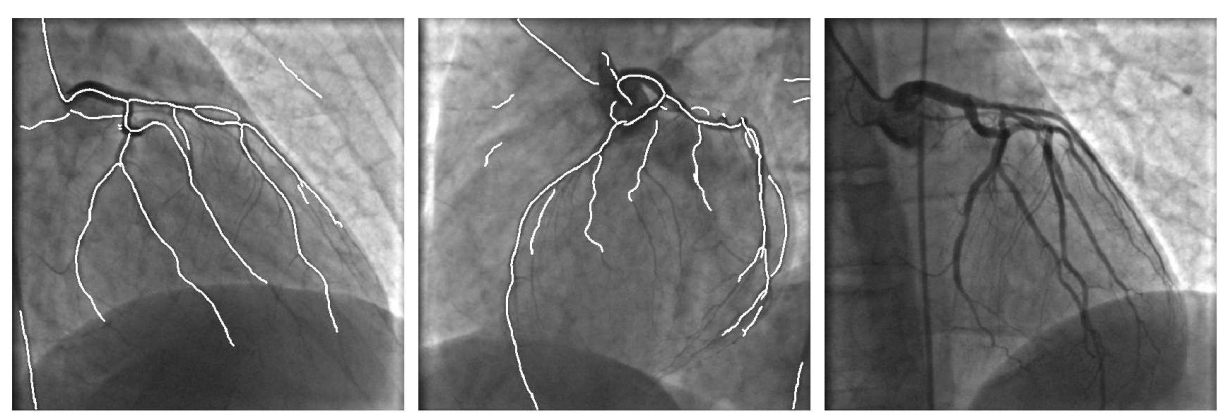

Fig. 4. Results of the dynamic programming based matching process. Centerlines in the first reference image are matched with centerlines in the second reference image (images in top row) according to the information contained in the two additional views (last images in top row). Projection of the 3$\mathrm{D}$ reconstructed points is presented in all reference images (bottom row).

in other reference images than as disconnected pieces. Exceptions may occur in case of superimposition or defective centerlines extraction. This constraint is called geometrical coherence.

Let $\left(p_{1}, q_{1}\right)$ and $\left(p_{2}, q_{2}\right)$ denote two matching pairs, where $p_{2}$ follows $p_{1}$ in a set of linked points in the extracted centerline in $I_{1}$. We define the penalty for two successive matching point pairs as a function of the distance $\left\|q_{1} q_{2}\right\|$ :

$$
\begin{gathered}
A M_{\text {Int }}\left(\left(p_{1}, q_{1}\right),\left(p_{2}, q_{2}\right)\right)=\rho\left(\left\|q_{1} q_{2}\right\|\right) \\
\text { with } \rho(d)=\left\{\begin{array}{lll}
0, & \text { if } & d \leq d_{l} \\
\frac{d-d_{l}}{d_{h}-d_{l}}, & \text { if } & d_{l}<d<d_{h} \\
1, & \text { else }
\end{array}\right.
\end{gathered}
$$

Thresholds $d_{l}$ and $d_{h}$ are chosen such that matched points whose distance is below $d_{l}$ are not penalized, and such that the ones whose distance is above $d_{h}$ are not overpenalized since they may indicate a discontinuity in the matching points sequence. Typically, $d_{l}=2$ pixels and $d_{h}=50$ pixels for $512^{2}$ images.

$\triangleright$ Matching criterion: Finally, the criterion measuring the quality of a matching configuration $\left(p_{i}, q_{i}\right)_{i=1 \ldots N}$ is chosen to be the weighted sum of the external energy term for all points pairs and of the internal energy term for all successive points pairs:

$$
\begin{aligned}
& A M\left(\left(p_{i}, q_{i}\right)_{i=1 \ldots N}\right)=-\sum_{i=1 \ldots N} A M_{\mathrm{Ext}}\left(p_{i}, q_{i}\right) \\
& \quad+\alpha \sum_{i=1 \ldots N-1} A M_{\mathrm{Int}}\left(\left(p_{i}, q_{i}\right),\left(p_{i+1}, q_{i+1}\right)\right) .
\end{aligned}
$$

The optimal set of correspondences, $\left\{\hat{q}_{1}, \ldots, \hat{q}_{N}\right\}=$ $\arg \min A M\left(\left(p_{i}, q_{i}\right)_{i=1 \ldots N}\right)$, is computed by a dynamic programming based approach [23], which enables to find the global optimum in predictable time and low computational complexity. We denote by $A \hat{M}\left(C, \mathcal{C}_{I_{2}}\right)=$ $\hat{A M}\left(\left(p_{1}, \ldots, p_{N}\right), \mathcal{C}_{I_{2}}\right)=A M\left(\left(p_{i}, \hat{q}_{i}\right)\right)$ the minimal value of the asymmetric matching criterion.

This optimization is repeated for all sets of linked points of image $I_{1}$. Figure 4 shows an example of results provided by matching process. The sum of the above matching criterion over all the sets of linked points in $I_{1}$ is assumed to be a quality measure of the 3-D reconstruction of all vessel centerlines: it is defined by $\sum_{C \in \mathcal{C}_{I_{1}}} A \hat{M}\left(C, \mathcal{C}_{I_{2}}\right)$ and we call it the global asymmetric matching criterion. Note that this criterion depends on the projection matrices M (see Eq. (4)).

2) Matching symmetrization: the above described reconstruction method is intrinsically asymmetric. Centerlines in image $I_{1}$ are matched with centerlines in image $I_{2}$ according to the remaining images, and the result depends on the choice of both images $I_{1}$ and $I_{2}$ which is undesirable.

A symmetric reconstruction is then achieved by considering all ordered pairs of images among the reference images. With our notations, we end up considering $\tilde{\mathcal{R}}(\tilde{\mathcal{R}}-1)$ pairs of images. This yields a number of 3-D reconstructed centerlines. The ones that are supposed to represent the same 3-D vessel may be slightly shifted from each other, mainly because of the respiratory motion, but also of an imperfect synchronization of reference images and of geometrical reconstruction errors.

Figure 5 shows the result of this symmetric reconstruction. The global matching criterion after symmetrization is merely given by the sum of all global asymmetric matching criteria over all ordered pairs of reference images, and is defined by

$$
\sum_{I_{1} \in \mathcal{R}} \sum_{I_{2} \in \mathcal{R} \backslash\left\{I_{1}\right\}} \sum_{C \in \mathcal{C}_{I_{1}}} A \hat{M}\left(C, \mathcal{C}_{I_{2}}\right) .
$$




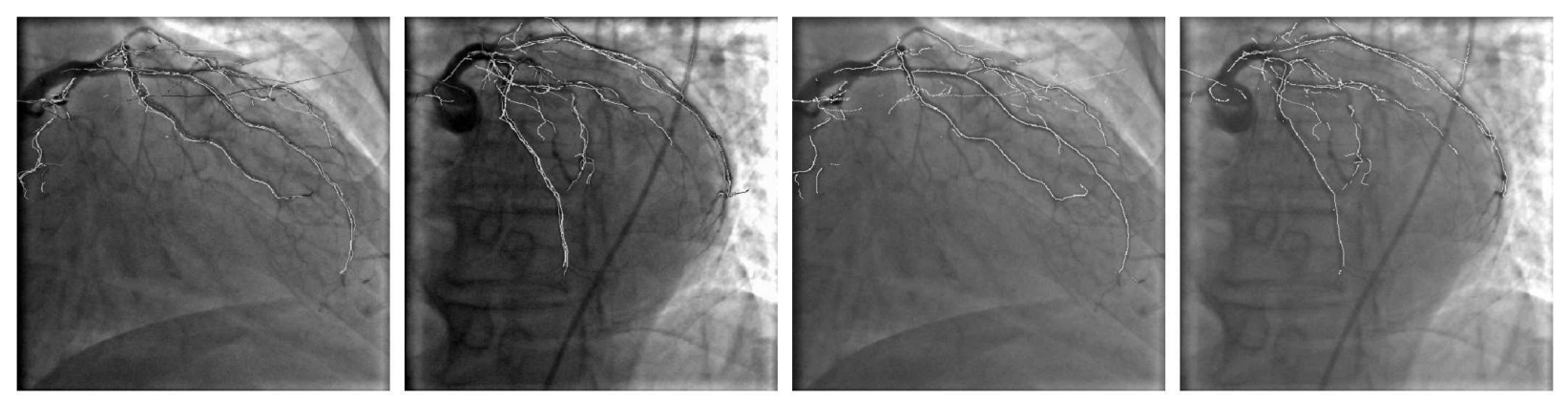

Fig. 5. Left, symmetric reconstruction: projection in two reference images of the centerlines reconstructed by considering all the ordered pairs of four reference images (a different greytone is associated with each asymmetric reconstruction), 3-D centerlines reconstructions appear redundant and slightly shifted. Right, fusion of the redundant 3-D reconstructed centerlines sets presented at left: the reconstructed centerlines after applying the fusion process are projected in two reference images. A geometrically average reconstruction has been built.

a) Respiratory motion compensation: as shown in [24][27], the respiratory motion effect on the myocardium, and thus on the coronary artery position, can be approximated by a 3-D translation, mainly in the axial direction. From the acquisition point of view, it corresponds to a translation of the images in their acquisition plane, that can be encoded in the calibration parameters, i.e. in the $\mathbf{M}_{n}$ matrices. Indeed, as demonstrated in [28], the global matching criterion reaches its minimum for optimal calibration parameters. We thus consider optimizing the global matching criterion with respect to the cameras translation in their acquisition plane, i.e.

$$
\begin{aligned}
& \left\{\hat{\mathbf{M}}_{i}\right\}_{i / I_{i} \in \mathcal{R}}=\arg \min _{\mathbf{M}_{i}} \sum_{I_{1} \in \mathcal{R}} \sum_{I_{2} \in \mathcal{R} \backslash\left\{I_{1}\right\}} \sum_{\left(p_{1}, \ldots, p_{N}\right) \in \mathcal{C}_{I_{1}}} R_{\left\{q_{j}\right\} \subset \mathcal{C}_{I_{2}}}\left[-\sum_{j=1 \ldots N} \frac{1}{\tilde{\mathcal{R}}-2} \sum_{I_{a} \in \mathcal{R} \backslash\left\{I_{1}, I_{2}\right\}} R_{a} M_{p_{j} q_{j}}\right) \\
& \left.\quad+\alpha \sum_{j=1 \ldots N-1} \rho\left(\left\|q_{j} q_{j+1}\right\|\right)\right]
\end{aligned}
$$

in order to estimate the respiratory motion. As a side result, we also obtain the 3-D reconstructed centerlines for the optimal projection matrices.

To optimize the global matching criterion, we use a FSQP optimization method implementation [29], [30]. It results in a translational correction in acquisition plane for reference images, reflecting respiratory motion effect. We propagate this information to other than reference images by linearly interpolating corrections that were found for the two surrounding reference frames.

3) Reconstruction fusion: the respiratory motion compensation improved the reconstruction of 3-D centerlines, particularly by reducing the slight shift observed between asymmetric reconstructions, but is not sufficient to yield a perfect superimposition of the 3-D centerlines obtained from the different asymmetric reconstruction. Keeping multiple reconstructions of the same 3-D vessels may bias the forthcoming 4-D motion estimation since it introduces spatial imprecision. Consequently, we fuse the distinct reconstructed centerlines sets in a single set where previously redundant points appear only once. We also store redundancy information, as it is a useful indicator for confidence in reconstructed points.

The fusion relies on a threshold representing the maximum shift distance allowed for redundant points. We set it to 5 millimeters which is an approximate value for largest observable coronary artery diameter. Two 3-D centerline sets are fused in a geometrical manner: for each point in the first set, we find the closest point in the second set, if their distance is smaller than a distance threshold, then points are considered redundant and are replaced by their barycenter, else the closest point is added as a new point. Iterating this process for all 3$\mathrm{D}$ reconstructed centerline sets leads to a fused 3-D centerline reconstruction. Figure 5 illustrates the effect of fusion on redundant 3-D reconstructed centerline sets.

Additionally, redundancy of fused points is given by the number of points that contributed to the fused point position. In practice, we build an application $\Lambda: \mathcal{M} \longrightarrow \mathbb{R}$, which associates the number of contributions $\Lambda(M)$ to any point $M$ in the fused 3-D centerlines set $\mathcal{M} . \Lambda(M)$ can be interpreted as the confidence value for the 3-D reconstructed point $M$.

At the end of this first stage, we have 3-D reconstructed centerlines, including confidence indices, that have been corrected from respiratory motion effect. This reconstruction was obtained from a few images from the same cardiac phase.

\section{4-D MOTION COMPUTATION}

From above computations, a 3-D reconstruction of the coronary artery centerlines at the cardiac reference time is obtained, as well as the respiratory motion compensation for all the rotational sequence images. This 3-D model will now be used to estimate the cardiac motion.

Contrary to 3-D motion approaches usually involved in the biplane case [2], [31], that estimate a 3-D motion from time to time, we use all frames simultaneously, independently from the cardiac phase at which they were acquired, to estimate a global motion, parameterized over space and time, hence called 4-D motion.

Contrary to Chen's approach [32] that requires a 3-D reconstruction at each cardiac cycle and the explicit establishment of correspondences between 3-D reconstructions to compute the 4-D motion, only one single 3-D reconstruction (at the reference cardiac cycle) is needed here, and the 4-D motion is inferred from image-based measures. 


\section{A. Motion parameterization}

To choose a motion parameterization, we recall some features of coronary artery motion: spatial and temporal smoothness and semi-local spatial and temporal influence.

These characteristics led us to choose a parameterization based on B-hypersolid, which is a 4-D tensor product of 1-D B-splines $B()$ [33]. This parameterization is a generalization of 3-D B-solids proposed in [34].

Extremal space coordinates are computed from the bounding box of the 3 -D centerlines set $\mathcal{M}$. The spatial support was sampled using control points spaced 2 centimeters apart, leading to typically 10 control points along each spatial dimension. Time extremal values were given by the interval $[0,1[$. This interval was sampled using 10 control points. In practice, we used cubic B-splines, providing a sufficient number of degrees of freedom. Knots vectors on space coordinates were chosen open uniform. This allowed one of the B-spline basis functions to be non null on the spatial bounds of the motion, and thus the motion may be non null on the spatial bounds of the motion support. On the opposite, knots vector in time coordinate was chosen uniform. This properties enforces that all B-spline basis functions to be null on the temporal bounds, and thus that the motion was constrained to be null at equivalent reference times $t=0$ and $t=1$.

Sets $\mathcal{I}, \mathcal{J}$, and $\mathcal{K}$ respectively discretize $x, y$, and $z$ space coordinates, set $\mathcal{L}$ discretizes time coordinate. Their respective cardinals are $\tilde{\mathcal{I}}, \tilde{\mathcal{J}}, \tilde{\mathcal{K}}$, and $\tilde{\mathcal{L}}$. Under a B-hypersolid motion $\Phi$ : $\mathbb{R}^{p} \times \mathbb{R}^{3} \times \mathbb{R} \longrightarrow \mathbb{R}^{3}$, parameterized by vector $\mathbf{p} \in \mathbb{R}^{p}$ (the knot points coordinates), the position of point $M=(x, y, z)$ after the application of the displacement, evaluated at normalized time $t$, is given by:

$$
\begin{gathered}
\Phi(\mathbf{p}, M, t)=\quad M+\sum_{i, j, k, l} B_{i}(x) B_{j}(y) B_{k}(z) B_{l}(t) \mathbf{p}_{i j k l} \\
\text { with } \mathbf{p}_{i j k l} \in \mathbb{R}^{3} \quad \forall i, j, k, l .
\end{gathered}
$$

\section{B. Motion optimization}

Estimating the heart motion now boils down to finding the optimal parameter vector $\hat{\mathbf{p}}$ that will exhibit the best coherence with the 2-D displacements observed in images $I_{n}$. This is achieved through the optimization of a criterion that aims at quantitatively evaluating the coherence of a B-hypersolid motion $\Phi(\mathbf{p}, . .$.$) with the angiogram sequence, through an$ external energy term, and that penalizes degenerate motions, through a regularization term.

The multiscale filter response, $R_{n}^{\star}$, is used as the likelihood that a pixel belongs to an artery projection. Summing the values of these responses for the projected 3-D reconstructed points $M$ under motion gives us the external energy term $\Psi: \mathbb{R}^{p} \longmapsto \mathbb{R}$ of the criterion:

$$
\begin{gathered}
\Psi(\mathbf{p})=\frac{1}{\tilde{\mathcal{N}} \tilde{\Lambda}} \sum_{n \in \mathcal{N}} \sum_{M \in \mathcal{M}} \Lambda(M) R_{n}^{\star}\left(\hat{\mathbf{M}}_{n}\left(\Phi\left(\mathbf{p}, M, t_{n}\right)\right)\right) \\
\text { with } \tilde{\Lambda}=\sum_{M \in \mathcal{M}} \Lambda(M) .
\end{gathered}
$$

Note that the multiscale filter response of projected point $M$ is weighted by its reconstruction confidence $\Lambda(M)$.
Successive steps for the evaluation of external energy term of the criterion are then:

- motion application $\Phi: \mathbb{R}^{p} \times \mathbb{R}^{3} \times \mathbb{R} \longmapsto \mathbb{R}^{3}$,

- projection application $\hat{\mathbf{M}}_{n}: \mathbb{R}^{3} \longmapsto \mathbb{R}^{2}$,

- multiscale filter response value $R_{n}^{\star}: \mathbb{R}^{2} \longmapsto \mathbb{R}$, and

- weighting by confidence index $\Lambda: \mathcal{M} \subset \mathbb{R}^{3} \longrightarrow \mathbb{R}$.

To prevent degenerated optimal motions, we add three internal energy terms which penalize motions with large amplitude, motions with erratic spatial behavior, and motions with erratic temporal behavior.

To estimate the motion amplitude, we evaluate the normalized sum over the control points of the square norm of vectors $\mathbf{p}_{i j k l}$ :

$$
\Gamma_{1}(\mathbf{p})=\frac{1}{\tilde{\mathcal{I}} \tilde{\mathcal{J}} \tilde{\mathcal{K}} \tilde{\mathcal{L}}} \sum_{i, j, k, l}\left\|\mathbf{p}_{i j k l}\right\|^{2} .
$$

To estimate the motion smoothness, we evaluate the normalized sum over the control points of the square norm of the vector difference between $\mathbf{p}_{i j k l}$ and its spatial neighbors $\mathcal{V}_{\mathbb{R}^{3}}\left(\mathbf{p}_{i j k l}\right)$ (in terms of 26-connectivity in 3-D) and its temporal neighbors $\mathcal{V}_{T}\left(\mathbf{p}_{i j k l}\right)$ (in terms of 2-connectivity in 1D):

$$
\begin{aligned}
& \Gamma_{2}(\mathbf{p})=\frac{1}{\tilde{\mathcal{I}} \tilde{\mathcal{J}} \tilde{\mathcal{K}} \tilde{\mathcal{L}}} \sum_{i, j, k, l} \frac{1}{\tilde{\mathcal{V}}_{\mathbb{R}^{3}}\left(\mathbf{p}_{i j k l}\right)} \sum_{\mathbf{p}_{i^{\prime} j^{\prime} k^{\prime} l} \in \mathcal{V}_{\mathbb{R}^{3}}\left(\mathbf{p}_{i j k l}\right)}\left\|\mathbf{p}_{i j k l}-\mathbf{p}_{i^{\prime} j^{\prime} k^{\prime} l}\right\|^{2} \\
& \Gamma_{3}(\mathbf{p})=\frac{1}{\tilde{\mathcal{I}} \tilde{\mathcal{J}} \tilde{\mathcal{K}} \tilde{\mathcal{L}}} \sum_{i, j, k, l} \frac{1}{\tilde{\mathcal{V}}_{T}\left(\mathbf{p}_{i j k l}\right)} \sum_{\mathbf{p}_{i j k l^{\prime}} \in \mathcal{V}_{T}\left(\mathbf{p}_{i j k l}\right)}\left\|\mathbf{p}_{i j k l}-\mathbf{p}_{i j k l^{\prime}}\right\|^{2}
\end{aligned}
$$

where $\tilde{\mathcal{V}}_{\mathbb{R}^{3}}\left(\mathbf{p}_{i j k l}\right)$ and $\tilde{\mathcal{V}}_{T}\left(\mathbf{p}_{i j k l}\right)$ are the respective cardinals of sets $\mathcal{V}_{\mathbb{R}^{3}}\left(\mathbf{p}_{i j k l}\right)$ and $\mathcal{V}_{T}\left(\mathbf{p}_{i j k l}\right)$.

The final criterion for 4-D motion optimization is:

$$
\Upsilon(\mathbf{p})=\Psi(\mathbf{p})-\alpha_{1} \Gamma_{1}(\mathbf{p})-\alpha_{2} \Gamma_{2}(\mathbf{p})-\alpha_{3} \Gamma_{3}(\mathbf{p}) .
$$

The knot vectors properties and discretization scheme lead to approximately 10 degrees of freedom along each coordinate, a degree of freedom being a three-dimensional vector. Thus, vector $\mathbf{p}$ typically has 30000 components. Consequently, optimizing the criterion requires a method dedicated to very large scale nonlinear optimization problems. It can be noticed that the four terms of $\Upsilon$ can be analytically derived. For instance, the gradient of $\Psi$ is given below:

$$
\frac{\partial \Psi(\mathbf{p})}{\partial \mathbf{p}}=\frac{1}{\tilde{\mathcal{N}} \tilde{\Lambda}} \sum_{n, M} \Lambda(M) \frac{\partial R_{n}^{\star}}{\partial \mathbf{M}_{n}} \frac{\partial \hat{\mathbf{M}}_{n}}{\partial \Phi} \frac{\partial \Phi\left(\mathbf{p}, M, t_{n}\right)}{\partial \mathbf{p}}
$$

As an optimization procedure, we thus chose the Polak-Ribière variant of the nonlinear conjugate gradient algorithm [35] and used the CONMIN implementation described in [36]. The initial motion is set to null $(\mathbf{p}=\mathbf{0})$. Optimization process leads to an optimal parameterization $\hat{\mathbf{p}}$ and associated optimal motion $\Phi(\hat{\mathbf{p}}, .,):. \mathbb{R}^{3} \times \mathbb{R} \longrightarrow \mathbb{R}^{3}$ that will thereafter simply be denoted by $\Phi$. The motion $\Phi(., t): \mathbb{R}^{3} \longrightarrow \mathbb{R}^{3}$ for a given normalized time $t$ is denoted $\Phi_{t}$. Figure 6 presents the results for motion computation by comparing 3-D centerlines projection before and after 4-D motion application. 


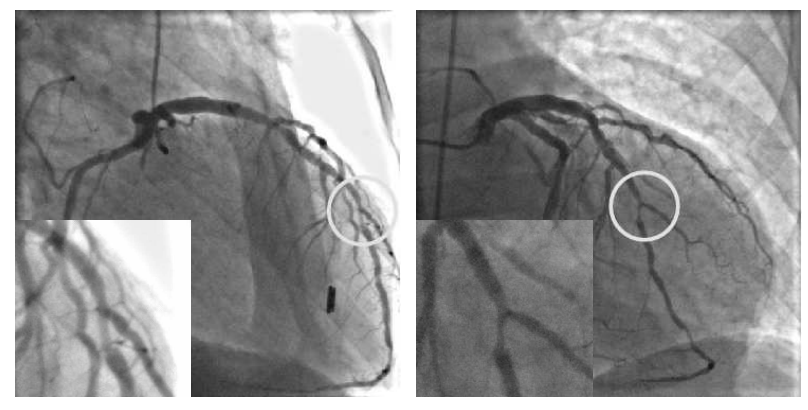

Fig. 8. Stabilized display of a stenosis. Two images that were acquired at distinct cardiac phases, from distinct viewing angles, in which we manually pointed the moving region of interest (a stenosis located at a bifurcation). Bottom left in images: focused and automatically centered images around the region of interest in the same images.
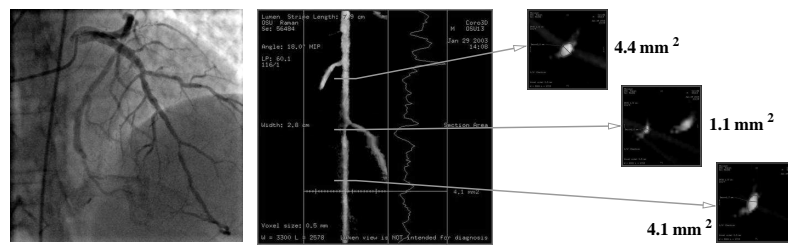

Fig. 9. Left: original angiogram. Right: vessel cross-sections measures (before, at, and after a stenosis).

coronary arteries is not straightforward, since the catheter may have a more simpler motion.

Obviously, such assertions have to be verified through clinical validation studies. Before initiating them, a retrospective analysis of our experiments is currently conducted.

The presented results and computation times have been obtained with the angiograph acquisitions being subsampled (from $768 \times 768$ to $512 \times 512$ pixels), for computational purposes. Additional experiments, conducted with full resolution images, did not show any visual difference.

At this point, we systematically use the end-diastole images as reference images to reconstruct a 3-D centerline model. In the future, it would be interesting to automatically select the most stable period of the cardiac cycle, which is the appropriate period for 3-D reconstruction, and that may depend on the heart rate.

We remark that the quality of the reconstruction (assessed visually) is directly correlated to the number of cardiac cycles that can be used for the processing. Typically, a number of 4 cardiac cycles (this corresponds to 5 reference times, i.e. $\tilde{\mathcal{R}}=5$ ) yields a visually good reconstruction, while artifacts or errors (for instance, in the centerline reconstruction) are more likely to occur when only 3 cardiac cycles are usable. The latter situation mainly arises because of a poor synchronization between rotation and contrast agent injection. Such defects happened for the first acquisitions, when the physicians did not master the rotational acquisition, but not for the last ones because of a fast learning curve [13]. It suggests that the quality of the reconstructions will increase for the future experiments.

We observe that the computational time of the reconstruction of a 3-D centerline model represents about one third of the total execution time. This is due to the re-estimation of the camera parameters, i.e. the respiratory motion (modeled as a translation) estimation. Since the acquisition duration is about a few seconds, it is possible to ask for a breath hold during the acquisition. This way, not only the total reconstruction time will be greatly decreased, but we will make sure to avoid the additional problems due to the respiratory motion estimation.

The roots of the coronary arteries are difficult to reconstruct since they are perpendicular to the rotation axis. This particular point is going to be investigated in relation with clinical partners.

It turns out that the first improvements will come from the design of an other acquisition protocol. Then, some quantitative quality measures have to be defined to assess both the overall quality of the reconstruction and all the different steps of the method (now excluding the respiratory motion estimation). This will help to optimize each step separately (e.g. the fusion of the centerline reconstructions). An other improvement in the future could be to automatically identify not only the heart cycles as we do now but also to identify the most appropriate period in the heart cycle for applying the multi-ocular algorithm. From the algorithm perspective, it has to be the most stable period along the heart cycle.

\section{CONCLUSION}

We presented a novel and stand-alone method to successively produce a 3-D reconstruction of coronary artery centerlines, a 4-D motion estimation of coronary arteries, and a 3-D tomographic reconstruction of coronary arteries from one single rotational X-ray acquisition. In contrast to other approaches reported in the literature, we are able to use almost all of the acquired frames, thank to the estimation of the coronary arteries motion.

Experiments conducted on real clinical data produced visually good reconstructions which demonstrate the practicability of such an approach. A further analysis encourages us to define a different acquisition protocol (with a breath hold) to suppress artifacts due to the respiratory estimation. Future work will consist of a clinical evaluation of the method, and will encompass both a quantitative assessment of the quality of the reconstruction and the identification of methodological improvements.

\section{ACKNOWLEDGMENTS}

We gratefully thanks clinicians (Doctors S.V. Raman, R.D. Magorien, and C.A. Bush from Ohio State University, at Columbus, United States of America, and Doctor W.R. Rüdiger Simon from Universtaetsklinikum, at Kiel, Germany). The authors thank Frédéric Devernay, from INRIA Rhône-Alpes, for fruitful discussions and technical knowledge sharing.

\section{REFERENCES}

[1] J. Mackay and G. A. Mensah, Eds., The Atlas of Heart Disease and Stroke. World Health Organization, 2004. [Online]. Available: http://www.who.int/cardiovascular_diseases/resources/atlas/en/

[2] S. Ruan, A. Bruno, and J. Coatrieux, "3D motion and reconstruction of coronary arteries from biplane cineangiography," Image and Vision Computing, vol. 12, no. 10, pp. 683-689, 1994. 
[3] A. Wahle, H. Oswald, and E. Fleck, "3D heart-vessel reconstruction from biplane angiograms," IEEE Computer Graphics and Applications, vol. 16, no. 1, pp. 65-73, 1996.

[4] S. Y. Chen and C. E. Metz, "Improved determination of biplane imaging geometry from two projection images and its application to threedimensional reconstruction of coronary arterial trees," Med Phys, vol. 24, no. 5, pp. 633-54, 1997.

[5] S. J. Chen and J. D. Carroll, "3-d reconstruction of coronary arterial tree to optimize angiographic visualization," IEEE Trans Med Imaging, vol. 19, no. 4, pp. 318-36, 2000.

[6] S. Raman, R. Magorien, R. Vaillant, P. Payne, K. Benali, and C. Bush, "Rotational Cardiovascular X-Ray Imaging for Left Coronary Artery Angiography Using a Digital Flat-Panel Cardiac Imaging System," American Journal of Cardiology, vol. 90, no. 6A, 2002.

[7] E. Kuon, P. Niederst, and J. Dahm, "Usefulness of Rotational Spin for Coronary Angiography in Patients with Advanced Renal Insuffi ciency," American Journal of Cardiology, vol. 90, no. 4, pp. 369-373, 2002.

[8] V. Rasche, M. Grass, R. Koppe, A. Bücker, R. Günther, H. Kühl, J. Op de Beek, R. Bertrams, and R. Suurmond, "ECG-gated 3D rotational coronary angiography," in Proceedings of Computer Assisted Radiology and Surgery. Paris: Springer-Verlag, 2002, pp. 826-831.

[9] O. Wink, R. Kemkers, S.-Y. J. Chen, S. Chen, and J. D. Carroll, "Intraprocedural coronary intervention planning using hybrid 3-dimensional reconstruction techniques," Academic Radiology, vol. 10, no. 12, pp. 1433-41, 2003.

[10] B. Movassaghi, V. Rasche, M. Grass, M. A. Viergever, and W. J. Niessen, "A quantitative analysis of 3-d coronary modeling from two or more projection images," IEEE Trans Med Imaging, vol. 23, no. 12, pp. 151731, 2004.

[11] S. Bonnet, A. Koenig, S. Roux, P. Hugonnard, R. Guillemaud, and P. Grangeat, "Dynamic X-ray computed tomography," Proceedings of the IEEE, vol. 91, no. 10, pp. 1574-1587, 2003, emerging Medical Imaging Technology.

[12] C. Blondel, R. Vaillant, G. Malandain, and N. Ayache, "3D tomographic reconstruction of coronary arteries using a precomputed 4D motion fi eld," Physics in Medicine and Biology, vol. 49, no. 11, pp. 2197-2208, 2004, special Issue: Seventh International Meeting on Fully 3D Image Reconstruction in Radiology and Nuclear Medicine.

[13] S. V. Raman, R. Morford, M. Neff, T. T. Attar, G. Kukielka, R. D. Magorien, and C. A. Bush, "Rotational X-ray coronary angiography," Catheterization and Cardiovascular Interventions, vol. 63, no. 2, pp. 201-7, 2004

[14] J. Serra, Image analysis and mathematical morphology. Academic Press, 1982.

[15] A. Frangi, W. Niessen, K. Vincken, and M. Viergever, "Multiscale Vessel Enhancement Filtering," in Proceedings of Medical Image Computing \& Computer Assisted Intervention (MICCAI) 1998, ser. LNCS, W. Wells, A. Colchester, and S. Delp, Eds., vol. 1496. Springer-Verlag, 1998, pp. 130-137.

[16] Y. Sato, S. Nakajima, N. Shiraga, H. Atsumi, S. Toshida, T. Koller, G. Gerig, and R. Kikinis, "3D multi-scale line fi lter for segmentation and vizualisation of curvilinear structures in medical images," Medical Image Analysis, vol. 2, no. 2, pp. 143-168, 1998.

[17] K. Krissian, G. Malandain, N. Ayache, R. Vaillant, and Y. Trousset, "Model-based detection of tubular structures in 3D images," Computer Vision and Image Understanding, vol. 80, no. 2, pp. 130-171, 2000.

[18] C. Lorenz, I. Carlsen, T. Buzug, C. Fassnacht, and J. Weese, "Multiscale line segmentation with automatic estimation of width, contrast and tangential direction in 2D and 3D medical images," in Proceedings of CVRMed-MRCAS, ser. LNCS, J. Troccaz, W. Grimson, and R. Mösges, Eds., vol. 1205. Grenoble: Springer-Verlag, 1997, pp. 233-242.

[19] T. Lindeberg, Scale-Space Theory in Computer Vision. Dordrecht, Netherlands: Kluwer Academic Publishers, 1994.

[20] G. Giraudon, "An Effi cient Edge Following Algorithm," in Proceedings of 5th Scandinavian Conference on Image Analysis, vol. 2, Stockholm, 1987 , pp. $547-554$.

[21] A. Merle, G. Finet, J. Liénard, and I. Magnin, "3D reconstruction of deformable coronary tree skeleton from two X-ray angiographic views," in Proceedings of Computers in Cardiology. Cleveland, Ohio: IEEE, 1998, pp. 775-760.

[22] C. Blondel, R. Vaillant, F. Devernay, G. Malandain, and N. Ayache, "Automatic Trinocular 3D Reconstruction of Coronary Artery Centerlines from Rotational X-ray Angiography," in Proceedings of Computer Assisted Radiology and Surgery (CARS). Paris: Springer-Verlag, 2002, pp. 832-837.

[23] E. Dijkstra, "A note on two problems in connection with graphs," Numerische Mathematik, vol. 1, pp. 269-271, 1959. Kinematics and the Implications for the spatial resolution in coronary imaging," Magnetic Resonance in Medicine, vol. 33, no. 5, pp. 713-719, 1995.

[25] A. Holland, J. Goldfarb, and R. Edelman, "Diaphragmatic and cardiac motion during suspended breathing: Preliminary experience and implications for breath-hold MR imaging," Radiology, vol. 209, no. 2, pp. 483-489, 1998.

[26] P. Danias, M. Stuber, R. Botnar, K. Kissinger, R. Edelman, and W. Manning, "Relationship between motion of coronary arteries and diaphragm during free breathing: Lessons from real-time MR imaging," American Journal of Roentgenology, vol. 172, no. 4, pp. 1061-1065, 1999.

[27] J. Keegan, P. Gatehouse, G. Yang, and D. Firmin, "Coronary artery motion with the respiratory cycle during breath-holding and freebreathing: implications for slice-followed coronary artery imaging," Magnetic Resonance in Medicine, vol. 47, no. 3, pp. 476-481, 2002.

[28] C. Blondel, G. Malandain, R. Vaillant, F. Devernay, E. Coste-Manière, and N. Ayache, "4D Tomographic Representation of Coronary Arteries From One Rotational X-Ray Sequence," in Proceedings of Medical Image Computing \& Computer Assisted Intervention (MICCAI), ser. LNCS, vol. 2878. Montreal: Springer-Verlag, 2003, pp. 416-423.

[29] C. Lawrence, J. Zhou, and A. Tits, User's Guide for CFSQP Version 2.5: A C Code for Solving (Large Scale) Constrained Nonlinear (Minimax) Optimization Problems, Generating Iterates Satisfying All Inequality Constraints, University of Maryland, 1997. [Online]. Available: http://www.aemdesign.com/download-cfsqp/cfsqp-manual.pdf

[30] C. Lawrence and A. Tits, "A Computationally Effi cient Feasible Sequential Quadratic Programming Algorithm," SIAM Journal on Optimization, vol. 11, no. 4, pp. 1092-1118, 2001.

[31] G. Shechter, F. Devernay, A. Quyyumi, E. Coste-Manière, and E. McVeigh, "Three-Dimensional Motion Tracking of Coronary Arteries in Biplane Cineangiograms," IEEE Transactions in Medical Imaging, vol. 22, no. 4, pp. 493-603, 2003.

[32] S. Y. J. Chen, S. Chen, and J. D. Carroll, "Kinematic and deformation analysis of 4-d coronary arterial trees reconstructed from cine angiograms," IEEE Trans Med Imaging, vol. 22, no. 6, pp. 710-21, 2003.

[33] M. Unser, "Splines: A perfect fit for signal and image processing," IEEE Signal Processing Magazine, vol. 16, no. 6, pp. 22-38, 1999, IEEE Signal Processing Society's 2000 Magazine Award. [Online]. Available: http://bigwww.epflch/publications/unser9902.html

[34] P. Radeva, A. Amini, and J. Huang, "Deformable B-solids and implicit snakes for 3D localization and tracking of SPAMM MRI data," Computer Vision and Image Understanding, vol. 66, no. 2, pp. 163-178, 1997.

[35] P. Gill, W. Murray, and M. Wright, Practical optimization. Academic Press, 1982.

[36] G. Vanderplaats, CONMIN - A ForTran program for constrained function minimization - User's manual, NASA, 1978, technical Memorandum X-62282. [Online]. Available: http://mdob.larc.nasa.gov/mdo.test/CONMIN/manual.html

[37] J. Hsieh, E. Chao, J. Thibault, B. Grekowicz, A. Horst, S. McOlash, and T. J. Myers, "A novel reconstruction algorithm to extend the ct scan fi eld-of-view," Med Phys, vol. 31, no. 9, pp. 2385-91, 2004.

[38] B. Movassaghi and V. Rasche, "3D coronary reconstruction from calibrated motion-compensated 2D projections based on semi-automated feature point detection," in Proceedings of SPIE Medical Imaging: Image Processing, Newport Beach, CA, 2004.

[39] G. Herman, Image reconstruction from projections. Academic Press, 1980.

[40] A. Geist, A. Beguelin, J. Dongarra, W. Jiang, R. Manchek, and V. Sunderam, "PVM 3 user's guide and reference manual," Oak Ridge National Laboratory, Tech. Rep. ORNL/TM-12187, 1993.

[41] C. Blondel, G. Malandain, R. Vaillant, and N. Ayache, "Reconstruction of coronary arteries from one rotational X-ray projection sequence," INRIA, Research report 5214, 2004. [Online]. Available: $h$ ttp://www.inria.fr/rrt/rr-5214.html

[42] T. Sato, T. Araki, and M. Hanayama, "A viewpoint determination system for stenosis diagnosis and quantifi cation in coronary angiographic image acquisition," IEEE Transactions on Medical Imaging, vol. 17, no. 1, pp. 121-137, 1998.

[43] C. Blondel, G. Malandain, R. Vaillant, and N. Ayache, "4D Deformation Field of Coronary Arteries from Monoplane Rotational X-Ray Angiography," in Proceedings of Computer Assisted Radiology and Surgery (CARS), ser. International Congress Series, vol. 1256. London: Elsevier, 2003, pp. 1073-1078. 